\title{
Edipo, Ninetto, Davide: cecità e colpa tra Morante e Pasolini
}

\author{
Marco A. BAZZOCCHI ${ }^{1}$ \\ Università di Bologna \\ marco.bazzocchi@unibo.it
}

\begin{abstract}
RIASSUNTO
L'articolo intende analizzare i fitti e complessi rapporti di scambio artistico e creativo fra Pier Paolo Pasolini ed Elsa Morante. A partire dal mito di Edipo e dal diverso trattamento che i due autori ne danno rispettivamente nel film Edipo Re e nella pièce teatrale La serata a Colono, vengono analizzate affinità e differenze fra i due autori nella trattazione del concetto di colpa. L'analisi di questo concetto viene fatta discutendo la presenza della traccia edipica nell'opera di entrambi gli autori - nel caso di Morante è presa in analisi soprattutto la figura di Davide ne La Storia - e viene definita una relazione fra colpa e cecità.
\end{abstract}

Parole chiave: Pasolini, Morante, mito di Edipo, colpa, cecità.

Oedipus, Ninetto, Davide:

Sin and Blindness between Morante and Pasolini

\begin{abstract}
The article tries to analyze the dense and complex artistic relations between Pier Paolo Pasolini and Elsa Morante. Starting from the different representations the Oedipus myth in the film Edipo Re by Pier Paolo Pasolini and in the drama La serata a Colono by Elsa Morante, the article analyzes affinities and differences between Morante and Pasolini in the definition of the concept of guilt. The analysis of guilt takes place as a discussion of the presence of the Myth of Oedipus in both authors. In the case of Morante the analysis is focused on the character of Davide in La Storia; in this way it is determined a relation between guilt and blindness.
\end{abstract}

Key words: Pasolini, Morante, Myth of Oedipus, Sin, Blindnes.

${ }^{1}$ Dipartimento di Filologia Classica e Italianistica, via Zamboni 32, I-40126, Bologna (Bo), Italia. 
Edipo re di Pasolini è un'opera che trasgredisce sotto molti aspetti il modello sofocleo, come ha dimostrato Massimo Fusillo nel suo libro su Pasolini e la cultura greca. $\mathrm{Al}$ di là dei particolari, potremmo in sintesi dire che gli elementi più espliciti della trasgressione sono: 1. la resa infantile (aggressiva, incerta, lamentosa) del personaggio principale, 2. il ruolo di Giocasta, individuata esplicitamente come la portatrice di un sapere assoluto, 3. la figura di Tiresia, che ha le caratteristiche di un indovino ma anche di un poeta, con il quale Edipo si misura costantemente, 4. la sostituzione della figura di Antigone con la figura di Angelo, un ragazzo gioioso e dall'apparenza inconsapevole interpretato da Ninetto Davoli. La critica americana ha visto in quest'ultimo punto una esplicita forzatura omosessuale del testo tragico: un ragazzo adolescente al posto della figlia del re, in particolare un ragazzo legato sentimentalmente a Pasolini stesso. Credo che questo sia un effetto di lettura semplificante e banalizzante. Nella sceneggiatura, che a volte dice molte cose più delle immagini, Nunzio viene descritto con una funzione specifica: è il mediatore tra la città e Edipo, colui che riesce a avvicinare Edipo anche dopo che il sacer, cioè la maledizione divina, lo ha colpito. In quanto mediatore, e pur con un ruolo non centrale, Nunzio compie molti atti che hanno un valore profondamente simbolico. Quando Edipo si mostra con gli occhi deturpati di fronte alla città, è il "ragazzonunzio" l'unico che può accostarlo: in lui ci sono i segnali della semplicità, dell'innocenza e soprattutto della "pietà":

Ed ecco venire verso di lui il ragazzo-nunzio, con la sua umile faccia pietosa: tiene in mano qualcosa, però. È un flauto. Un flauto come quello di Tiresia. Il flauto di chi è cieco. Il flauto che fa tornare le cose nelle regole, che codifica lo scandalo. / Il ragazzo abituato agli umili servizi, non ha paura di avvicinare quell'uomo ridotto così male, con quelle piaghe ancora fresche e sanguinanti. Gli si avvicina, e gli allunga il flauto che Edipo non vede, ignora. / Allora il ragazzo gli prende una mano, e gliela mette sul flauto: la mano di Edipo cerca, riconosce. Stringe il flauto e, sorretto dal ragazzo, si incammina con lui. / I due se ne vanno - in quel silenzio eccessivo e sgradevole - mentre la folla li segue con gli sguardi, fatta estranea (Pasolini 2001: 1047-1048).

Per Vernant (Vernant 2001), Edipo oscilla tra due condizioni: quella del re divino e quella del pharmakos, il capro espiatorio. Ma va sottolineato che Edipo sta "tra" queste due condizioni, in un luogo speciale, indefinibile, e in questo luogo si apre lo spazio vero del tragico, quello che non consente definizione né parola riconosciuta. Nunzio e il flauto hanno la funzione di rendere rappresentabile questo spazio, cioè la transizione tra norma e sacro: Nunzio fa da mediatore tra Edipo e il mondo, il flauto rappresenta lo strumento con cui lo scandalo può ritornare a essere comunicazione, se pur comunicazione poetica.

L'operazione di Pasolini diventa più esplicita se la misuriamo con quella, di poco seguente ma dialogante, della Serata a Colono di Elsa Morante (il film viene presentato a Venezia il 3 settembre del 1967, e in contemporanea esce per Garzanti la sceneggiatura, mentre il Mondo della Morante è del 1968, Pasolini scrive un pezzo sul Caos il 27 agosto del '68, poi la lunga recensione poetica che esce in 
anteprima su Paragone dell'aprile 1969, e infine entra in Trasumanar e organizzar). L'intreccio di elementi che legano il testo della Morante al film di Pasolini non è ancora ben stato definito: innanzitutto direi che là dove Pasolini ha infantilizzato Edipo, la Morante ne ha al contrario sottolineato l'aspetto intellettuale e visionario, che emerge dalla particolare testualità dei suoi monologhi, infarciti di citazioni e di strutturazioni poetiche. La figura di Antigone viene di nuovo riportata ad un dominio femminile, anche se Morante gioca con il nome della figlia che esplicitamente dichiara di chiamarsi Ninetta («ce ne siamo partiti da casa di notte all'impruviso / che lui nun m'ha lasciato il tempo di pigliare nemmanco una borza o una valiscia che lui ma detto Ninetta andiamo andiamo» (Morante 1995: 41)), cioè si tratta di Ninetto/Nunzio che adesso assume veste femminile ma comunque mantiene le caratteristiche di ignoranza, ingenuità, popolarità del Ninetto reale ${ }^{2}$. Non penso - e cercherò di dimostrarlo con l'analisi che segue - che si possa ridurre questo passaggio a una semplice questione di genere ma credo sia necessario riferirsi a qualcosa di più profondo che tocca la concezione del personaggio dei due autori, o perlomeno il modo con cui il personaggio è portatore di una visione del mondo proprio nel momento in cui ha un corrispettivo nel mondo reale. Se pensiamo ai rimandi interni alla raccolta, possiamo ipotizzare che Antigone annunci la figura che troviamo poi al centro del Mondo, cioè il Pazzariello. Ma complicando ancora il gioco dei rimandi, e guardando a quanto dichiara a tutte lettere lo stesso Pasolini nella sua recensione poetica, il Pazzariello è a sua volta una anticipazione "figurale" del Ninetto Davoli reale:

Dunque, il Pazzariello io l'avevo già letto

(e conosciuto) prima che fosse nella tua opera politecnica.

La metodologia per approssimazione mimetica e un po' piaggiatrice

dei tuoi versi non ancora scritti,

aveva già infatti prodotto in me e nel mondo l'idea di Ninetto.

Te la devo. Te la devo (Pasolini 2003: II, 45).

Con la recensione Pasolini vuole infatti creare una serie di parallelismi tra lui e la Morante, con allusioni spesso criptiche al loro rapporto di amicizia, e uno dei momenti chiave è quello in cui Ninetto viene evocato come un Pazzariello in carne e ossa, dotato di quella carica erotica e fisica che invece - secondo Pasolini - il Pazzariello della Morante non possiede («La tua Summa pazzariello si è esaurita,

2 Per M. Fusillo (1996: 66), esiste «consonanza profonda» tra l'Antigone «dolce e popolare» della Morante e il «Ninetto giocoso» del film pasoliniano.

${ }^{3}$ Uso "figurale" nel senso auerbachiano che anche Pasolini utilizza spesso intendendo il rapporto tra una figura di finzione che completa una figura reale, la rende cioè più vera e reale. Ma per Pasolini a volte il rapporto si dà in direzione opposta, cioè è la figura reale che completa quella di finzione. 
dunque, prima e dopo il libro: / il suo luogo centrale, deputato (nel libro), devo dirlo, resta un po' vacante»).

Un segnale ulteriore è presente nel finale della Serata a Colono, quando la voce di Antigone-Ninetta urla disperatamente « $\mathrm{Pa}$ ', $\mathrm{Paaa}$ '» che è il termine dialettale di origine romanesca che rimanda al padre ma rimanda anche al nome Paolo. ${ }^{4}$ In un altro testo di Trasumanar (Uno dei tanti epiloghi) viene evocato un sogno dove Ninetto corre dietro a Pier Paolo che è in macchina e gli chiede « $A \mathrm{~Pa}^{\prime}$, mi porti con te? Me lo paghi il viaggio?» (Pasolini 2003: 101, corsivo nell'originale). Dunque l'appellativo «Pa» ha un valore ampio, dove il nome del Padre si coniuga con il nome di un padre che in realtà non può essere realmente padre, ma che adotta questo ruolo solo in un senso anomalo, fuori dalla regola, e solo eccezionalmente accetta la definizione di Padre. In una poesia sempre dedicata al rapporto con Ninetto, Pasolini analizza la nozione di "affetto" che si contrappone a quella di "amore" e dice che l'affetto provoca una tenerezza che impedisce sia di fecondare che di essere fecondati (il riferimento è a Socrate) eppure «si soccombe ad esso / con lo stesso senso di precipitare nel vuoto / che si prova gettando il seme, quando si muore / e si diventa padri» (Un effetto della vita, in: Pasolini 2003: 103). Questa morte connessa al diventare padri è ciò che Pasolini rifiuta sempre fino al momento in cui se ne rende consapevole a proposito del rapporto con Ninetto, che secondo lui rappresenta l'unica possibilità «di disfarsi senza dolore di se stessi». La paternità che sente verso Ninetto è una forma di "uscita" dal ruolo standardizzato di padre (non dimentichiamo che la Morante aveva già raccontato, nell' Isola di Arturo, l'avventura di un padre, Wilhelm Gerace, che si sottraeva, attraverso il desiderio omosessuale, al ruolo paterno, pur diventando nella fantasia del figlio un padre eroe).

Anche Edipo - per Morante - cerca disperatamente il modo di disfarsi di se stesso. Alla sua colpa sembra non poter esserci nessun rimedio, e sembra impossibile anche un secondo sacrificio, dopo quello degli occhi che in realtà ha iscritto una luce perpetua nel buio della notte di Edipo. Questa luce è la presenza del Padre, cioè del Sole Apollo, di fronte a cui - o contro cui - Edipo pronuncia i monologhi più straordinari dell'intero pezzo teatrale. Edipo è rimasto crocefisso (tracce della cultura cristiana attraversano i monologhi di Edipo, come ha notato Andrea Rodighiero) al punto eterno della sua colpa, cioè a Tebe: «Ma il punto del dolore continuo / che per le corde in estirpate dei tèndini m'inchioda al centro della ruota / è sempre là, uno, sempre / lo stesso: la città / della peste» (Morante 1995: 59). Morante chiude nella impossibile concentrazione di un unico punto tutta la vicenda di riscatto e purificazione di Edipo a Colono: quel punto implica l'impossibilità del tempo ma anche l'impossibilità del viaggio nello spazio che invece compare nelle battute di Ninetta/Antigone, capace di rievocare luoghi e

4 Anche A. Rodighiero (2007: 65, n. 35) rimanda all'uso romanesco con cui Pasolini veniva chiamato dagli amici. 
situazioni di un frammentato racconto in cui però il padre non si ritrova e che potrebbe essere solo frutto della fantasia infantile della figlia. L'Edipo della Morante è un errante nel delirio linguistico, non nello spazio. Ed è un intoccabile a cui neanche la figlia può portare sollievo, come invece fa Nunzio/Ninetto nel film di Pasolini. Per Vernant, Sofocle ha creato un raffinatissimo gioco di spostamenti nello spazio fuori da Atene per dare a Edipo la possibilità di trovare un luogo marginale, tra sacro e profano, da cui poter rivolgere il logos al coro. Questo avviene su un gradino scavato nella roccia, ai margini del bosco delle Eumenidi. Nella Morante, Edipo vede le Furie / Eumenidi come felini notturni «sante figlie dell'oscurità» «nictalope divine», ed è sotto la protezione di queste dee-gatte che Edipo può dare libero corso ai suoi deliri discorsivi.

C'è un altro elemento che però va individuato in questa doppia ripresa del personaggio di Edipo. Nel lungo e complesso discorso rivolto al Padre Sole, Edipo rivela caratteristiche fisiche che passano attraverso una catena di metafore vegetali e animali e che dissolvono la maschilità di Edipo, o per lo meno la modificano in direzioni che portano ai limiti della giurisdizione dell'umano. A questi limiti si trova la stessa Antigone, descritta dal padre come:

una zingarella semibarbara e di pelle scura come lui povera guagliuncella ma cresciuta per colpa della sua nascita, che in faccia ha i segni dolci e scostanti delle creature di mente un poco tardiva $[\ldots]$

L'adolescenza, la razza, la semplicità intellettuale sono i segnali che mettono Antigone al polo opposto del padre, poeta, profeta, uomo colto, ma la riportano a lui nella posizione di marginalità nei confronti dell'umano. Edipo e Antigone sono due forme viventi che non si possono riconoscere nella norma e che incarnano perfettamente la creaturalità da cui provengono i personaggi della Morante, quello spazio al limite del sociale, del razionale e del normale in cui si muovono tutti i personaggi dell'autrice, nei quali la mescolanza linguistica e somatica produce sempre zone di ibridazione e di indistinzione: incertezza di caratteri sessuali (Arturo, Manuele, Ninetta), indistinzione dei luoghi in cui avvengono le loro avventure (l'isola, il ghetto, l'ospedale, l'Andalusia ecc.), e infine instabilità delle loro condizioni psichiche (non a caso il monologo allucinato caratterizza spesso la forma del discorso dei personaggi). Questo spazio della mescolanza Agamben lo ha definito "parodia", intesa come esibizione di un codice letterario deviante rispetto alla norma, l'unico capace di dare rappresentazione dell'irrapresentabile (Agamben 2010: 120 e ss.). «Vattene, parodia» è la terribile ingiunzione con cui il giovane delinquente Stella allontana Wilhelm Gerace che lo sta corteggiando dall'esterno del carcere. Qui "parodia" indica appunto una deviazione dalla norma, ma è anche un termine retorico con cui si definisce un'opera che si sviluppa nella tangenza con un modello classico. Per questo possiamo considerare il termine con un valore ampio proposto da Agamben e estenderlo a molte zone dell'opera della scrittrice. Parodia potrebbe indicare quell'essere "a fianco" della norma, del discorso 
ufficiale, della razionalità da cui provengono in gran parte le creature del mondo morantiano. La Serata a Colono è parodia di Edipo a Colono di Sofocle, ma i suoi protagonisti sono in questo senso doppiamente parodici rispetto ai personaggi originari. $^{5}$

Pasolini non condivide con la Morante questa prospettiva di ibridazione del personaggio, però compie un'operazione sulla figura di Ninetto che forse risente della lettura del Mondo salvato. Nel 1968, subito dopo Edipo e Teorema, Pasolini gira un cortometraggio che ha come unico personaggio Ninetto e si intitola la Sequenza del fiore di carta. In questo corto di 9 minuti vediamo Ninetto camminare per le strade affollate di Roma, in mezzo a gente impegnata nel lavoro o in altre faccende. Ninetto si muove quasi danzando, senza pensieri, lungo via Nazionale, mentre in dissolvenza cominciano ad apparire scene di guerra e di morte, ma lui non viene distratto da niente proprio perché incarna la distrazione. Il suo camminaredanzare si scontra apertamente sia con la realtà degli altri sia con le cupe scene che interrompono per pochi attimi la sequenza principale. A un certo punto, una voce dal cielo chiama Ninetto e con insistenza pretende la sua attenzione. Ninetto non sente, continua nel suo ballo spensierato, esibendo addirittura in una mano un grande fiore rosso di carta. La voce di Dio lo incalza, e gli fa precise richieste: «Io voglio da te i frutti - i tuoi primi frutti. Frutti del tuo sapere e del tuo volere». Poi la voce sembra inquietarsi: «Tu sei innocente e chi è innocente non sa e chi non sa non vuole. Ma io che sono il tuo Dio ti ordino di sapere e di volere (qui parte in sottofondo la Passione secondo Matteo di Bach) A chi ha parlato Cristo? Agli innocenti perché sapessero! ascoltami! L'innocenza è una colpa! Gli innocenti saranno condannati perché non hanno diritto di esserlo! Io devo maledirli come il fico e farli morire». Ninetto è ora steso a terra, morto, accanto a lui il fiore rosso di carta. Pasolini ha sacrificato Ninetto, ma lo ha sacrificato proprio perché vuole dimostrare che anche gli innocenti diventano colpevoli nel momento in cui non accettano di divenire consapevoli del male che li circonda: Ninetto si sottrae alla voce di Dio (che nel film è composta di alcune voci diverse, tra cui quella di Pasolini, del regista Bertolucci, della nipote Graziella Chiarcossi) e viene allora punito, la sua innocenza si mostra fragile come un fiore di carta.

Così Pasolini interpreta la famosa parabola evangelica del fico punito da Cristo perché non produce frutti fuori stagione, quella stessa parabola che inquietava Simone Weil, secondo la quale nel Vangelo si intendeva la condizione di chi ha scarse doti naturali e quindi non è degno di doni spirituali. Pasolini inserisce la parabola nel contesto storico: se nel mondo c'è il male, gli innocenti non possono restar innocenti, devono prendere atto del male. Altrimenti il loro restare innocenti si ribalta in una colpa. E' interessante che Pasolini, subito dopo aver riconosciuto l'importanza dei F.P. nell'opera della Morante (i F.P. sono portatori della Grazia e della Carità), crei una specie di abiura a quel riconoscimento attraverso la ripresa

\footnotetext{
${ }^{5}$ Anche Concetta d'Angeli (2003: 120) insiste giustamente sul concetto di parodia.
} 
della parabola. Nel mondo contemporaneo gli innocenti non possono restare innocenti, dunque Ninetto viene sacrificato proprio perché porta su di sé quella Grazia che per Pasolini nelle poesie della Morante nasceva dall'Umorismo in quanto Carità, cioè in quanto dono puro. Qui Pasolini usa una citazione di San Paolo a cui ricorre spesso e vede nella terza virtù, dopo la Fede e la Speranza, la virtù migliore (méizon dè touton e agàpe): «Sì! L'UMORISMO COME CARITA', E' QUESTA ... LA GRAZIA!» (Pasolini 2003: 44).

Ma io credo che anche Morante avesse ben presente la parabola, e la avesse già utilizzata in filigrana (o parodia) nel monologo in cui Edipo racconta le metamorfosi che lo portano a crescere e a svilupparsi nel tentativo di donarsi in modo completo al Sole Padre Apollo. Qui infatti abbiamo una creatura arborea, un ulivo, che obbedisce in modo completo e indiscriminato alla legge del Padre. Anzi, è l'amore verso il Padre che muove fin dall'origine tutta l'esistenza:

Tutte le mie nascite innumerevoli sono state sotto il suo regno. E d'una in un'altra è per $L U I$ che mi sono incarnato in quest'ultima specie del dolore. Già fino dalle mie puerizie iniziali quando il mio corpo era un filo d'alga acquatica o una goccia dentro una conchiglia, c'era in me un'ALTRA ansia, che muoveva in cerca di LUI!

Quando Edipo racconta la parabola dell'ulivo (se posso ora chiamarla così) è la forza del desiderio a essere messa in scena come tensione che spinge una creatura fitomorfa ma indifferenziata («non sapevo se ero brutto o bello, e nemmeno / se ero una vergine o un fanciullo!») a crescere sotto l'influsso amoroso di una potenza che dall'esterno la spinge a modificarsi secondo una tensione erotica che ricalca da vicino la concezione di Spinoza sull'affectus, cioè sul passaggio che implica aumento e diminuzione di potenza in un corpo $^{6}$ :

E la linfa irrequieta scoppiava dalla mia corteccia

in lacerazioni e sfoghi simili a bruciature, screziando di un colore sanguigno / i miei frutti amarognoli. Era LUI

che dal cielo estivo autunnale

m'infiammava all'avventura straordinaria

percuotendomi con le sue fruste addenti e bagnandomi con la

sua saliva di miele e col suo seme radioso!

${ }^{6}$ Adatto al testo questa definizione di Deleuze sulla nozione di affectus in Spinoza (Deleuze 2007: 46): «l'affectus in Spinoza è una variazione continua (...) della forza di esistere, determinata dalle idee con cui entriamo in rapporto». 
Il corpo di Edipo-Ulivo cresce, cioè trova una forma, ubbidendo alla trazione da sé che viene dall'esterno: la sua è una metamorfosi attraverso la quale forme del non umano si integrano una nell'altra fino a portare a un risultato di pure potenzialità indistinte: «Ero un ibrido / dai ricci di capra e dai piccoli denti carnivori, / e avevo l'allegria della caccia: perché in ogni sangue / mi si faceva riconoscere LUI: colore e sapore». Potremmo ipotizzare che qui Edipo pronunci il discorso che avrebbe potuto pronunciare Arturo se avesse voluto tradurre in termini poetici e sintetici il suo rapporto con l'isola e con Wilhelm, senza distinguere razionalmente tra l'una e l'altro, vedendo nelle forme di vita dell'isola la manifestazione del potere del padre, e riconoscendo se stesso nell'insieme di quelle forme, fino al momento in cui la rottura con il padre non implica anche l'abbandono dell'isola (e, possiamo ipotizzare, l'adozione di una 'forma' definita, adulta, che però la Morante non ci fa vedere in quanto esterna al suo racconto).

Edipo è un personaggio che esce continuamente dai propri limiti, un personaggio in "estasi" e come tale possiamo considerarlo, all'interno della specificità del codice poetico, un concentrato di molti personaggi morantiani. A Edipo manca però l'occasione del sacrificio, così come gli manca quella della pacificazione attraverso il "passaggio" che caratterizza l'Edipo a Colono sofocleo. Tanto che la sparizione finale di Edipo, già più volte interpretata, avviene attraverso il rimando alla scala colorata della discesa agli inferi della dea Istar $^{7}$, con la quale Edipo si libera gradualmente della propria ansia di separazione dal divino, cioè dal LUI-Padre, che alla fine, con i versi di Hölderlin, domina come "Sacro Essere" nella scena vuota: Edipo sparisce, come la nuvola che passa sulla luna, e lascia dietro di sé solo l'urlo di Antigone che richiama il padre, ma un padre terreno, non un padre divino, dal momento che lei può vedere senza dolore nelle cose del mondo. E infatti sarà Antigone/Ninetta a diventare poi il Pazzariello e a reincarnarsi in tutte le figure di vera Grazia della seconda parte del libro: la Mutria, che assiste con coraggio al sacrificio di Cristo, e poi il Pazzariello, e poi la Carlottina.

Con la ritualità che deriva dal modello mitico, Morante ha dato a Ninetta la forza di generare una vera e propria genealogia di personaggi che possono sostenere in modo non tragico il rapporto col sacro. Così Morante risponde sotterraneamente a Pasolini, alla sua ansia di non poter essere "santo", alla sua omosessualità che non diventa perfezione e spesso produce pulsioni di annientamento. C'è un'altra strada, sembra dire Elsa, e questa strada passerà attraverso la grande opera realistica del sacrificio, la Storia, e attraverso la grande opera visionaria del sacrificio, Aracoeli. Ma questa strada implica un personaggio impuro, ibrido, indefinito come Antigone, cioè un personaggio che sa mettersi in rapporto col male senza doverne necessariamente riscattare la colpa, un "puro folle" che, al contrario del Ninetto sacrificato nel Fiore di carta, non è innocente ma nello stesso tempo non ha implicazioni dirette col male. Conosce il male che lo circonda ma ne resta

${ }^{7}$ Si veda in particolare il commento di Concetta d'Angeli (2003). 
incontaminato, oppure, come nel caso di Emanuele in Aracoeli, sa andare fino in fondo all'origine del male (il corpo materno) per riscoprire una sopravvivenza d'amore nella figura paterna.

Malgrado questo, nella Storia Morante ha voluto lasciare una presenza del sacrificio edipico, cioè di un personaggio che ha subito una violenza all'origine e non se ne può liberare se non con un processo di autodistruzione. Così il delirio della colpa e della distruzione del proprio sé nella sterilità del sacrificio è incarnata da Davide, che costituisce un anello di congiunzione necessario tra Useppe e Nino. Il sistema è complesso, e va analizzato con attenzione proprio perché non credo ci sia niente di casuale in questa costruzione. Pasolini, che scrisse una recensione piena di riserve, si era reso conto che l'operazione è tanto anomala da non poter essere ricondotta a fatti letterari ma parlava di una vera e propria visione filosofica che si distribuisce nelle zone del romanzo e crea un effetto di passaggi continui tra forze vitali. Come se il romanzo fosse un organismo che viene amputato progressivamente di arti, o segmenti del proprio corpo, ma trova il modo di esistere spingendo le forze vitali nelle zone corporee che rimangono e che si caricano di nuova energia. Solo che per essere il più possibile antimorantiano (nella recensione) Pasolini adotta un metodo di vivisezione razionale che sembra inaridire la costruzione e ridurla a pezzi monchi dell'insieme. Pasolini aveva già in precedenza detto che Morante linguisticamente mette in rapporto la grammatica con lo spirito, e questo era il suo modo per mettere a fuoco quel particolare rapporto del mondo che prima ho definito estatico, per cui i personaggi sono sempre nel mondo ma in loro si aprono improvvisamente vie di fuga o mentali o fisiche che li portano fuori dal mondo, a contatto con livelli di esperienza che non possono essere contenuti nelle codificazioni del mondo reale. Edipo è il modello di questo fatto. Nella Storia, tutti vivono questa esperienza: Ida, che scopre nella sua umiltà un rapporto inconscio con le radici del male, Useppe, che raccoglie su di sé il male pur restandone incorrotto, e Davide, che è segnato dal male, anzi da quello che viene definito dal narratore uno "stupro", cioè un rapporto violento con il sacro di cui Davide viene segnato fin dall'infanzia e di cui porta il segnale nel volto fin da quando appare nello stanzone dei Mille (anche per questo la gatta Rossella, come le Eumenidi feline della Notte a Colono, è l'unica che sente per lui vera attrazione, ma anche in lei come sappiamo c'è una forma deviata di rapporto col mondo che si manifesta nel ripudio della maternità):

Adesso invece la sua fisionomia era segnata da qualcosa di corrotto, che ne pervertiva $i$ lineamenti dall'interno. E questi segni, ancora intrisi di uno stupore terribile, parevano prodotti non da una maturazione graduale; ma da una violenza fulminea, simile a uno stupro (Morante 1995: 199).

Questo segno della diversità, che poi viene analizzato narrativamente in più punti del racconto, manifesta il modo in cui l'innocenza di Davide è diventata una colpa. Non è un caso che sia proprio lui, in osteria, a raccontare la parabola del fico, 
e l'albero maledetto ricompare nel suo delirio fino a che non avviene una identificazione con esso:

Si racconta che un cristo (non importa quale, era un cristo) una volta camminando per una via di campagna ebbe fame e andò per cogliere un frutto da un albero di fico. Ma siccome non era stagione, l'albero non aveva frutti: nient'altro che foglie incommestibili ... E allora Cristo lo maledisse, dannandolo alla sterilità perpetua ... Il senso è chiaro: per chi riconosce Cristo al suo passaggio, è sempre stagione. E chi non riconoscendolo gli nega la propria frutta col pretesto del tempo e della stagione, è maledetto (Morante 1995: 591).

Finché il segno della violenza si ribalta in nuova violenza agita: «In questo sogno, l'albero maledetto (che stavolta è chiaramente lui stesso, Davide) non è solo un traditore della rivoluzione vera, un violento nato e un assassino, ma anche uno stupratore». Dunque la forza della colpa si è manifestata in Davide con la stessa deviazione che lo ha colpito. Da essere violentato Davide diventa essere violento, da aggredito aggressore. $E$ in questo Nino non lo può capire, mentre Useppe sente in lui la presenza del male esattamente come la sente nelle fotografie dei deportati a Auschwitz. Per questo, quando Useppe si reca nella casa di Davide ormai moribondo e viene da lui cacciato, la scena della disperazione del bambino ricalca la scena dello sconvolgimento cosmico che accompagna la morte di Cristo sulla croce: «Di certo in quel momento la terra non ha tremato; ma Useppe ebbe la medesima esatta sensazione di un terremoto che erompeva dal centro dell'universo» (Morante 1995: 618).

Pasolini sembra non riconoscere questo aspetto della personalità di Davide e spostando la sua attenzione su un dato stilistico - condanna il personaggio in quanto carente di una dimensione di credibilità realistica:

...il parlato di Davide - scrive Pasolini - non ha riscontro in nulla: il ragazzo si presenta come bolognese, in realtà è mantovano, ma parla una specie di veneto. Non c'è tuttavia angolo dell' Alta Italia in cui cadere si dica cader. Per ogni dove, là, nell'Alta Italia, è cascare che ha trionfato eliminando ogni altra forma concorrente. Che Davide dica cader è offensivo per il lettore: ma è soprattutto offensivo per lui. Dov'è il così grande amore della Morante per lui, se essa è poi così pigra da non fare il minimo sforzo per ascoltare come parla? (Pasolini 1999: 2101)

Il giudizio è particolarmente severo, soprattutto perché Pasolini accusa Morante di essere stata una madre "cattiva", cioè di aver creato un figlio (un personaggio) senza amarlo veramente, e quindi trascurandolo nel momento in cui gli ha dato la parola. Ma Pasolini non può non essersi accorto che ancora una volta la prospettiva materna di Morante ha prodotto un personaggio in cui c'è anche lui stesso: Davide in lotta contro la famiglia borghese, Davide che vuol capire gli operai e entra in fabbrica, Davide che si autodistrugge perché non sa come liberarsi di una colpa che lo marchia a fuoco è anche Pier Paolo-Edipo, a cui si è aggiunta la passione algida 
di Simone Weil a spostare il baricentro del personaggio verso quella discesa autodistruttiva che invece Pasolini non avrebbe mai ammesso. Dunque, negando autenticità a Davide, e insinuando il dubbio di una sua creazione "in difetto di amore", Pasolini allontana da sé il personaggio, ne rifiuta ogni similarità e fraternità. E soprattutto gli nega appropriatezza linguistica che equivale a dire mancanza d'amore per la realtà.

Anche nei confronti di Nino-Ninnuzzo-Ninnarieddu Pasolini sembra non voler vedere, e accusa il personaggio di essere falso «tutto costruito aprioristicamente»e diventare «stupendamente reale» solo nel momento della morte. Questo nuovo Pazzariello in carne e ossa, che riprende ancora una volta alcune caratteristiche di Ninetto Davoli, è il mediatore necessario tra il mondo e tutti gli altri personaggi, non per niente abita fuori dal romanzo, o meglio ai confini del romanzo, e non al suo interno: l'allusione che viene fatta al dio Mercurio quando conduce per la prima volta Useppe fuori di casa, è un'allusione fondamentale. Come Mercurio Nino non può essere definito in nessuno dei tanti aspetti in cui compare, ma sta al confine tra tutti, è l'incarnazione della leggerezza vera del Pazzariello, e anche per questo muore ma senza che il male lo abbia sfiorato, a differenza di Useppe, che vede il male, lo sente, lo assorbe ma ne resta incontaminato, Nino non lo vede neanche: di fronte alla colpa che si incarna in Davide, lui ride e continua ad amare Davide come un fratello. Così Nino e Davide si pongono ai poli opposti di un medesimo arco esistenziale. L'amore irriflesso di Nino per la vita ha lo stesso peso delle elucubrazioni intellettuali di Davide: nella concezione di Morante la realtà è composta dal loro insieme, non c'è distinzione. Per Pasolini invece la semplicità di Edipo (del suo Edipo bambino) è solo la premessa a una forma di conoscenza che deve trasformarsi in consapevolezza dolorosa e quindi sublimarsi produzione poetica: non a caso il "trasumanar" dantesco non impedisce, secondo l'insegnamento di San Paolo, il passaggio all' "organizzar". Anche Ninetto infatti deve diventare consapevole, se vuole salvarsi, deve cioè ascoltare la voce di Dio, di un Padre imperioso che gli ordina di conoscere. Morante non accetta questa linea di distinzione maschile, tanto è vero che la sua Antigone è una ragazza ignorante del popolo che possiede come unica forma di consolazione la varietà del racconto fantastico e la bizzarria della lingua. Dentro questo stesso dispositivo fantastico vivono Ida e Useppe, uniti nella incapacità di conoscere e nell'unica virtù del sentire: sentono il male, non lo conoscono. Per questo Pasolini, alla fine della sua recensione alla Storia, rievoca il Mondo salvato come opera in cui si incarna perfettamente l'ideologia morantiana, mentre quella stessa ideologia, trasformata in organismo narrativo, dunque in personaggi, «perde ogni credibilità» e diventa «un fragile pretesto» che mette in crisi la stessa «sproporzionata macchina narrativa» che ha messo in moto. Il realismo è per Pasolini un paradigma con cui attraversare il romanzo ma nello stesso tempo per non vedere (o censurare) il nuovo ordine con cui Morante riesce a tenere legati il male e la colpa, innestando una parabola evangelica sul corpo del mito di Edipo e creando la triplice figurazione maschile: Nino, Useppe, Davide. Il fico maledetto e il tiranno colpevole di uno stupro rivivono nel giovane intellettuale che sacrifica se stesso per liberarsi di una colpa 
iscritta nella carne. Così la Storia apriva una strada che avrebbe portato verso Aracoeli, un altro racconto di espiazione del dolore spinto al di là del paradigma mitico. Questa espiazione passava attraverso un rapporto non delimitato alla discendenza maschile o a quella femminile. Come recentemente ha dimostrato Manuele Gragnolati (2013), proprio Morante è capace di aprire una zona di ibridazione che va al di là delle separazioni o delle categorizzazioni imposte dall'ordine simbolico nelle costruzioni culturali ${ }^{8}$. Questi segnali, Morante li aveva disseminati nel romanzo cercando di coinvolgere, attraverso figure che risalgono al Pazzariello/Antigone, un fratello anomalo come Pasolini. Ma Pasolini non ha voluto vedere. Come Edipo, è rimasto cieco di fronte alla realtà.

\section{RIFERIMENTI BIBLIOGRAFICI}

AgAmben, Giorgio (2010) Categorie italiane. Studi di poetica e di letteratura, Roma-Bari, Laterza.

D’ANGeli, Concetta (2003) Leggere Elsa Morante. Aracoeli, La Storia e Il mondo salvato dai ragazzini, Roma, Carocci.

DeleuZE, Gilles (2007) Cosa può un corpo? Lezioni su Spinoza, Verona, Ombre corte.

Gragnolati, Manuele (2013) Amor che move. Linguaggi del corpo e forme del desiderio in Dante, Pasolini e Morante, Milano, Il Saggiatore.

Fusillo, Massimo (1996) La Grecia secondo Pasolini. Mito e cinema, Firenze, La Nuova Italia.

MORANTE, Elsa (1995) Il mondo salvato dai ragazzini, Torino, Einaudi.

MORANTE, Elsa (1995) La Storia, Torino, Einaudi.

PASOLINI, Pier Paolo (1999) Recensione a La storia di Elsa Morante, in Saggi sulla letteratura e sull'arte, Milano, Mondadori, 1999, p. 2101. [Originariamente appare in Descrizioni di descrizioni]

PASOLINI, Pier Paolo (2001) Per il cinema, a cura di Walter Siti e Franco Zabagli, Milano, Mondadori, pp. 1047-1048.

Pasolini, Pier Paolo (2003) Tutte le poesie, a cura di Walter Siti, Milano, Mondadori.

Rodighero, Andrea (2007) Una serata a Colono. Fortuna del secondo Edipo, Verona, Edizioni Fiorini.

VERNANT, Jean-Paul (2001) Edipo tra due città, in Jean-Paul Vernant, Pierre VidalNaquet, Mito e tragedia due. Da Edipo a Dioniso, Torino, Einaudi, pp. 161-196.

\footnotetext{
8 Più precisamente, Gragnolati si riferisce ad Aracoeli e indica "una soggettività nonedipica e in progress, non ancora fissata secondo le norme e - anziché sentita come imperfetta - ora abbracciata nella sua fluidità, singolarità e parzialità proprio come possibilità di contrapporsi alle opposizioni binarie" (Gragnolati: 2013: 160).
} 